\title{
Pratiques
}

Linguistique, littérature, didactique

$177-178$ | 2018

Langage oral à l'école maternelle. Étude d'un corpus

homogène

\section{Apprendre à parler en justifiant ses dires collectivement pour comprendre un récit lu ou raconté à l'école maternelle : propos des élèves et interventions de l'enseignant}

Learning to talk by justifying ideas together in order to understand a story in kindergarten: students' statements and teacher's mediation

Glaís Sales Cordeiro

\section{(2) OpenEdition}

\section{Édition électronique}

URL : http://journals.openedition.org/pratiques/4060

DOI : 10.4000/pratiques.4060

ISSN : 2425-2042

Éditeur

Centre de recherche sur les médiations (CREM)

\section{Référence électronique}

Glaís Sales Cordeiro, «Apprendre à parler en justifiant ses dires collectivement pour comprendre un récit lu ou raconté à l'école maternelle : propos des élèves et interventions de l'enseignant », Pratiques [En ligne], 177-178 | 2018, mis en ligne le 20 juillet 2018, consulté le 20 avril 2019. URL : http:// journals.openedition.org/pratiques/4060 ; DOI : 10.4000/pratiques.4060

Ce document a été généré automatiquement le 20 avril 2019.

(C) Tous droits réservés 


\title{
Apprendre à parler en justifiant ses dires collectivement pour comprendre un récit lu ou raconté à l'école maternelle : propos des élèves et interventions de l'enseignant
}

Learning to talk by justifying ideas together in order to understand a story in kindergarten: students' statements and teacher's mediation

\author{
Glaís Sales Cordeiro
}

\section{Introduction}

1 Les enjeux du colloque Apprentissage du langage oral à l'école maternelle. Regards croisés sur un corpus homogène étaient directement liés à l'importance du langage oral en début de scolarité dans des situations didactiques, des «séances de langage», sur la compréhension d'un récit, lu ou raconté par un enseignant, provenant d'un album de la littérature de jeunesse. Dans cette contribution, nous essayons de montrer comment les élèves apprennent à parler sur (et de) ce récit pour le comprendre dans le cadre de ces situations, qui impliquent la construction d'une pensée et d'un parler collectifs sous le guidage de l'enseignant.

2 Nous commençons par esquisser quelques éléments de définition concernant les conceptions de l'oral et de son enseignement-apprentissage à l'école maternelle afin de mieux déterminer le positionnement théorique assumé en vue de l'analyse du corpus de « séances de langage " proposé. Nous essayons ensuite de caractériser les spécificités des interactions verbales en maternelle lorsque les élèves et l'enseignant parlent sur et des 
textes lus ou racontés en nous référant également à la perspective théorique adoptée dans nos propres travaux, depuis laquelle nous examinerons les dialogues didactiques présents dans les séances constituant le corpus. Nous poursuivons avec la présentation de la démarche méthodologique adoptée et de quelques exemples d'analyse. Nous concluons avec des remarques sur la portée didactique des démarches de justification collectives pour apprendre à parler sur un récit dans le but de le comprendre et le statut du dialogue scolaire à l'école maternelle.

\section{Enseigner et apprendre à parler à l'école maternelle : différentes approches et différents points de vue}

L'oral a toujours été omniprésent dans les classes et ceci à tous les niveaux de la scolarité. Il constitue un des moyens les plus importants pour la socialisation des élèves et la transmission de savoirs scolaires, surtout à l'école maternelle. Toutefois, les approches de l'oral et de son enseignement peuvent être aussi diverses que leurs objectifs, comme le montre très bien le texte de cadrage du colloque (voir la présentation du numéro).

En ce qui concerne, plus précisément, les pratiques de l'oral dans les classes maternelles après le mouvement de rénovation de l'enseignement du français, celles-ci peuvent viser la correction syntaxique des énoncés des jeunes élèves (Lentin, 1972), le développement des capacités de ces derniers à parler ensemble dans des "groupes conversationnels » (Florin, 1995) ou, à partir des années 90, l'apprentissage de capacités langagières ancrées dans des situations de communication variées.

5 Deux sous-ensembles caractérisent ces approches plus récentes de l'oral. Dans le premier sous-ensemble, la parole devient un objet d'enseignement et d'apprentissage à part entière. Il s'agit d'apprendre à produire des textes oraux à travers des séquences d'enseignement qui instaurent l'oral comme objet d'étude à travers des activités d'observation, de pratique simplifiée, de réflexions sur les spécificités langagières des genres de textes produits dans des situations de communication (voir Thévenaz-Christen, 2005). Dans le deuxième sous-ensemble, l'oral est un médiateur, un moyen d'enseignement. Dans ce cas, le dialogue scolaire permet de rendre présent, à travers les interactions enseignant-élève(s) et entre élèves, un contenu disciplinaire (lié à une discipline scolaire comme les sciences, les arts, etc.) et/ou langagier (des conduites discursives) pour que toute la classe se les approprie. En ce sens, l'écoute active, compréhensive, interprétative et les types d'étayage fournis par l'enseignant constituent un enjeu majeur dans les situations didactiques.

6 Les objectifs de ces approches plus contemporaines de l'oral étant l'apprentissage de nouveaux modes de parler (le parler scolaire) et de penser, elles visent, à travers les situations didactiques proposées, la construction d'un rapport secondarisé au monde présupposant le développement des capacités réflexives inédites, non singulières et dépersonnalisées (collectif scolaire) chez les élèves, « c'est-à-dire des capacités générales d'identification, de distinction, de distanciation, de généralisation, d'anticipation et de contrôle» (ibid., p. 69). Ces modes de socialisation spécifiques constituent la «forme scolaire » (Vincent, Lahire \& Thin, 1994), « émergente » (Thévenaz-Christen, 2005) dans le contexte de l'école maternelle. Cette forme scolaire en devenir est caractérisée par une tension entre les contextes d'apprentissage plus spontanés de la petite enfance et des apprentissages « réactifs» (Vygotski, 1985) ou " provoqués » (Brossard, 2008) propres au 
cadre scolaire, comme nous essayerons de le montrer à travers l'analyse de quelques extraits du corpus sélectionnés pour cette contribution. Il en découle que l'école maternelle représente un passage entre les interactions «naturelles» orientées par des besoins communicatifs immédiats du quotidien (en famille et dans les institutions de la petite enfance également) et les pratiques langagières "artificielles", typiquement scolaires, visant des nouvelles conceptualisations (Vygotski, 1985).

7 Comme nous le verrons plus loin, l'analyse du corpus proposé dans le cadre du colloque suggère que dans les «séances de langage » mises en place, les enseignants cherchent, effectivement, à initier leurs jeunes élèves à des interactions orales ancrées dans la forme scolaire émergente dans le but de les faire discuter et réfléchir sur le texte lu ou raconté par l'enseignant. Ceci, afin d'expliciter, à travers les étayages proposés, les significations attribuées aux différents éléments constitutifs de l'histoire. En ce sens, le parler scolaire s'inscrit ici dans la logique d'une approche de l'oral où ce dernier est un vecteur, un moyen d'enseignement, visant d'autres apprentissages langagiers, en l'occurrence, la compréhension d'un récit. Afin de mieux délimiter les caractéristiques spécifiques de ce type de dialogue scolaire, examinons ce que disent les recherches à propos de l'activité de lecture d'albums et de textes à l'école maternelle.

\section{Apprendre à parler ensemble sur (et de) un texte lu par l'enseignant pour le comprendre}

8 Selon plusieurs auteurs, les interactions enseignant-élèves contribuent de façon décisive à la construction des significations concernant des textes lus ou racontés par l'enseignant à l'école maternelle. La notion de médiation devient centrale dans ce contexte, comme le soutenait, par exemple, F. Grossmann (1996) il y a une vingtaine d'années. En mettant en évidence le rôle médiateur de l'enseignant lors de la lecture partagée d'albums de la littérature de jeunesse avec de jeunes élèves, ce chercheur a souligné l'importance de la lecture expressive et dramatisée ("adaptation textuelle») par l'adulte et du dialogue autour du texte en lien avec l'observation des images et en réaction aux énoncés lus. Le programme d'enseignement conçu par M. Brigaudiot (2000) sur la construction des représentations de l'écrit et des capacités à lire et à écrire ainsi que les activités favorisant le développement des compétences transactionnelles chez des jeunes lecteurs proposées par S. Terwagne et M. Vanesse (2008) font des pratiques de lecture partagée un concept clé également. Par ailleurs, V. Boiron (2010) défend l'idée que, dans les classes maternelles, la lecture à voix haute dramatisée effectuée par l'enseignant ainsi que les « activités de contage » (Boiron, 2014) permettent aux élèves d'accéder aux significations du récit, aux sentiments et aux motivations des personnages, les activités de lire et de raconter un album s'instaurant dans le cadre d'une "communauté discursive", constituée par l'enseignant expert et les élèves apprentis, ce qui engendre des formes de " coopération interprétative ».

9 En consonance avec cet ensemble de recherches dont nous ne donnons ici que quelques exemples $^{1}$, nous avons cherché, pour notre part (Cordeiro et al., 2012; Cordeiro, 2014), à appréhender le dialogue didactique autour d'un album lu par l'enseignant comme un réseau collectif partagé de justifications qui permettent la construction par les élèves des significations qui y sont liées. En nous référant aux travaux d'É. Nonnon $(1999,2016)$, nous avons considéré l'activité de verbalisation comme un révélateur pour l'enseignant 
des processus de construction de nouvelles connaissances par les élèves présupposant, entre autres, la capacité à justifier leurs propres dires. Un outil au service de l'enseignement et des apprentissages, par conséquent.

10 Afin de mieux cerner la teneur des interactions verbales scolaires, nous nous sommes appuyée, plus spécifiquement, sur des études portant sur la fonction didactique des justifications. D'après ces recherches, les démarches de justification constituent pour l'enseignant un moyen d'accès aux raisonnements des élèves en lien avec l'objet enseigné, vu qu'elles impliquent une forme d'explicitation des propos par la présence d'éléments (la plupart du temps hiérarchisés) qui les étayent (Garcia-Debanc, 1996/1997). Lorsqu'il justifie, l'élève doit montrer à l'enseignant que son discours est "rationnellement acceptable » (Chartrand, 2000) et que sa réponse n'est pas aléatoire. Il doit affirmer et justifier ses dires par des marqueurs de relations (car, donc, puisque, alors) entre des « faits pertinents » et des notions élaborées dans le domaine étudié. Dûment transposés aux dialogues didactiques concernant la compréhension sur (et des) textes lus prenant place dans les classes maternelles, ces éléments peuvent fournir des indicateurs pour une double analyse de la fonction didactique médiatrice des démarches de justification : celle des justifications produites par les élèves pour étayer leurs propos en réponse aux questions posées par l'enseignant et celle du rôle médiateur de l'enseignant dans la construction collective avec les élèves des significations liées à l'album lu. Ces indicateurs constitueront donc un des piliers sur lesquels s'appuiera l'analyse des données issues du corpus CLEA que nous présenterons plus bas. Le deuxième pilier concerne la spécificité de l'objet enseigné dans les situations didactiques rassemblées dans le corpus, à savoir la compréhension d'un récit, lu ou raconté par l'enseignant, provenant d'un album de la littérature de jeunesse. C'est la raison pour laquelle nous présentons ensuite quelques éléments qui nous semblent incontournables pour la compréhension des récits.

\section{Le système récit-personnages comme une condition d'accès à la compréhension des récits}

11 La compréhension de textes écrits (et oraux) en maternelle étant tributaire des situations didactiques dans lesquelles les élèves peuvent/doivent justifier leurs raisonnements collectivement, il est également essentiel de délimiter les contours de l'objet sur lequel porte le dialogue didactique, sans quoi il devient difficile d'examiner les conditions d'accès à cet objet.

12 Pour comprendre des "récits élaborés » (Grossmann, 1996) ou "qui s'interprètent" (Terwagne \& Vanesse, 2008), le lecteur doit s'approprier les rapports dynamiques entre les sentiments éprouvés par les personnages et les raisons qui les amènent à agir de telle ou telle manière, la mise en intrigue, mais aussi percevoir la façon dont ils sont désignés et caractérisés par le narrateur. À travers leur "être », leur "dire " et leur "faire " (Tauveron, 1995), les personnages constituent une unité de structuration du récit (Reuter, 1988) régie par le texte (et les images, dans le cas des albums). Ils construisent la trame narrative tout en se construisant par elle à travers les différentes relations qu'ils établissent entre eux dans leur participation aux évènements et aux dialogues de l'histoire² (Tauveron, 1995; Adam \& Revaz, 1996). Tel est le cas du récit dans l'album Danger dans le potager, lu ou raconté aux élèves dans les « séances de langage » constituant le corpus CLEA. Grâce à son esprit intrépide, le personnage principal, Pierre lapin, 
s'aventure dans un lieu interdit, le jardin potager de Monsieur Grégory, le voisin, afin de déguster des délicieux mets. En se confrontant à son antagoniste, Pierre expérimente divers sentiments dans les différentes péripéties vécues dans ce lieu et également lors du retour à la maison auprès de sa maman et de ses frères.

13 D'un point de vue didactique, l'accès au système dialectique constitué par les buts et raisons d'agir des personnages, leurs sentiments et les actions qui en découlent dans Danger dans le potager devient ainsi un objet d'enseignement et d'apprentissage. Comme dit précédemment, à l'école maternelle, l'appropriation de ces éléments a lieu à travers une construction collective des significations du récit, ponctuée par les interventions de l'enseignant dans le cadre des interactions langagières entre lui et les élèves. Dès lors, le dialogue didactique, soutenu par le pointage (Boiron, 2010) et la discussion des traits pertinents décelés par les élèves et par l'enseignant à propos des représentations et motivations des personnages liées aux différents évènements du récit, permet aux élèves de suivre la trame narrative, une meilleure compréhension du récit pouvant ainsi être assurée. Cette perspective a, de ce fait, orienté notre regard dans la démarche méthodologique que nous avons entamée pour analyser les données issues du corpus proposé. Nous la présentons ci-après.

\section{Démarche méthodologique de prise de connaissance et d'analyse du corpus}

\section{Le texte lu ou raconté et discuté avec les élèves : analyse préalable du système récit-personnages}

14 Afin de pouvoir définir un parcours d'analyse et d'interprétation du corpus CLEA, nous avons commencé par prendre connaissance du texte oral ou écrit utilisé comme support commun aux « séances de langage » qui le composent. Nous nous sommes référée au texte Danger dans le potager. Une aventure de Pierre Lapin, issu de l'album de Beatrix Potter et transcrit dans un document PDF trouvé sur une page du site Internet de l'Académie de Nancy-Metz ${ }^{3}$.

En nous basant sur la notion de système récit-personnages préalablement présentée, nous avons essayé d'identifier dans le texte les rapports entre les motivations, les sentiments et les actions des personnages de Pierre, de Monsieur Grégory et de Maman Lapin afin de mieux appréhender la dynamique de ces rapports dans le cadre du récit qu'ils constituent. Dans Danger dans le potager, le narrateur désigne explicitement la motivation initiale de Pierre, sa curiosité «d'aller voir le potager interdit » de Monsieur Grégory malgré les avertissements de Maman Lapin, et, dans une certaine mesure, son envie de s'échapper pour rentrer à la maison («il se met à courir, dans tous les sens, sans pouvoir retrouver l'endroit par lequel il est entré »; "il cherche désespérément à sortir du potager $»$ ). Les sentiments éprouvés par le personnage principal sont également pointés tout au long des évènements (son courage, son plaisir, son insouciance, sa peur, son épuisement, son stress) prenant place dans ce lieu tellement convoité et, également, lors du retour au terrier auprès de sa maman (son effondrement, son bouleversement). C'est pourquoi nous avons décidé de nous centrer, pour notre analyse, sur les échanges entre les enseignants et les élèves autour du comportement de ce personnage dans le récit. 


\section{Les « séances de langage " (corpus), la délimitation de l'empan de l'analyse et la démarche entreprise}

Dans une première « lecture flottante » des douze « séances de langage » du corpus, nous avons rapidement identifié la présence de deux grandes phases de discussion dans sept de ces séances : une première phase, où les élèves reconstituent collectivement le récit à travers les questions et aides fournies par l'enseignant ; une deuxième phase, où il s'agit d'une part de parler sur les personnages et, notamment, sur le personnage principal de Pierre, et d'autre part d'émettre un jugement moral à propos de son attitude.

Nous avons choisi de nous pencher sur les extraits de la deuxième phase d'échanges portant plus spécifiquement sur le comportement de Pierre dans le but de repérer les éléments qui s'en dégageaient.

Le tableau 1 regroupe les séances retenues avec des informations concernant le niveau scolaire, le type de quartier où se situe l'école et le nombre d'élèves enregistrés par classe.

Tableau 1. Choix des séances analysées

\begin{tabular}{|l|l|l|l|l|l|l|l|}
\hline Séance & $\mathbf{2}$ & $\mathbf{3}$ & $\mathbf{4}$ & $\mathbf{7}$ & $\mathbf{9}$ & $\mathbf{1 0}$ & $\mathbf{1 1}$ \\
\hline Niveau & GS & MS & GS & MS & MS & GS & GS \\
\hline Quartier & ZEP & hétéro-gène & hétéro-gène & sensible & sensible & hétéro-gène & sensible \\
\hline Nombre d'élèves & 8 & 9 & 10 & 8 & 9 & 12 & 7 \\
\hline
\end{tabular}

Pour chacune des sept séances, nous avons d'abord dégagé les types de questions formulées par les enseignants afin de déterminer la nature de ce questionnement. Dans un deuxième temps, nous avons examiné les réponses des élèves et, plus particulièrement, la pertinence des traits relevés dans les justifications proposées et négociées dans les échanges collectifs avec l'enseignant, relatifs aux sentiments, aux raisons d'agir et aux actions de Pierre dans le déroulement du récit.

Pour l'analyse, nous nous sommes inspirée des critères développés dans nos propres recherches sur l'enseignement et l'apprentissage de la compréhension en lecture de récits dans des classes du premier degré avec des élèves de 4 à 9 ans (Cordeiro, 2014; Thévenaz-Christen \& Cordeiro, sous presse). Ces critères sont présentés dans le tableau 2.

Tableau 2. Types de justification et traits pertinents prélevés par les élèves

\begin{tabular}{|lr|l|}
\hline $\begin{array}{l}\text { Niveaux } \\
\text { justification }\end{array}$ & de & Traits pertinents \\
\hline $\begin{array}{l}\text { Absence } \\
\text { justification } \\
\text { partielle }\end{array}$ & très & $\begin{array}{l}\text { Pas ou peu de prise en compte des rapports entre la caractérisation du } \\
\text { personnage et la chronologie/logique de ses actions }\end{array}$ \\
\hline
\end{tabular}




\begin{tabular}{|l|l|}
\hline $\begin{array}{l}\text { Justification } \\
\text { partielle }\end{array}$ & $\begin{array}{l}\text { Prise en compte partielle des rapports entre la caractérisation du } \\
\text { personnage et la chronologie/logique de ses actions }\end{array}$ \\
\hline $\begin{array}{l}\text { Justification } \\
\text { presque complète }\end{array}$ & $\begin{array}{l}\text { Prémisses de construction systémique (sans focalisation sur un seul aspect) } \\
\text { les rapports entre la caractérisation du personnage et la chronologie/ } \\
\text { logive des actions avec prise en compte éventuelle des sentiments, } \\
\text { motivations et actions des autres personnages }\end{array}$ \\
\hline
\end{tabular}

De notre point de vue, l'analyse des justifications produites dans le cadre du dialogue didactique peut révéler, dans le même temps, les niveaux de compréhension des élèves concernant les rapports systémiques entre les sentiments, motivations et actions des personnages dans les différents moments d'un récit ainsi que les manières d'en parler, élèves et enseignant, contribuant à la (re)construction de ces rapports. Nous avons alors essayé de vérifier, pour chaque « séance de langage » sélectionnée, dans quelle mesure les justifications relatives à la caractérisation de Pierre et à ses actions montraient, dans le dialogue entre les élèves et l'enseignant, des traces de (re)construction du système récitpersonnages de Danger dans le potager pouvant ainsi contribuer à l'apprentissage d'un (nouveau) mode de parler (scolaire) visant un rapport plus distancié au récit.

\section{Parler ensemble sur le personnage principal et son comportement dans le récit : (re)construire collectivement le système récit-personnages en justifiant ses dires}

22 Nous présentons ci-après quelques résultats d'analyse des échanges entre l'enseignant et ses élèves sur le personnage de Pierre ayant lieu dans les séances de langage choisies. Nous commençons par des commentaires généraux concernant ces séances et passons ensuite à un examen plus détaillé de quelques extraits d'interaction enseignant-élèves.

\section{Les caractéristiques du dialogue didactique dans l'ensemble des " séances de langage » retenues}

En ce qui concerne le type de questionnement effectué par les enseignants dans les sept « séances de langage » choisies, nous avons identifié la présence de questions ouvertes présupposant un positionnement de la part des élèves par rapport à Pierre et à son comportement (ses motivations, ses actions, ses sentiments). Rappelons qu'aucune orientation spécifique n'avait été donnée sur le type de questions à poser aux élèves, ce qui a engendré une certaine hétérogénéité dans le corpus quant au nombre d'interventions des enseignants ainsi qu'à leur nature, comme on le verra par la suite. Le texte de cadrage du colloque mentionne seulement que «sur le plan pédagogique » il a été « suggéré aux maîtres de réduire de manière drastique leur vitesse d'intervention et pour cela d'interroger systématiquement les élèves ayant levé le doigt avant de relancer la question pour une nouvelle série de réponses [...] ». "Sur le plan des apprentissages ", les enseignants devraient adopter " un "format" de séance régulier [...] en reprenant un même canevas d'une séance à l'autre [...] » (voir la présentation du numéro). 
Le tableau 3 recense les questions posées par les enseignants dans chaque séance.

Tableau 3. Questions posées par les enseignants pour entamer la discussion sur Pierre et son comportement

\begin{tabular}{|l|l|}
\hline Séances & Question(s) posée(s) par chaque enseignant \\
\hline 2,9 & $\begin{array}{l}\text { qu'est-ce que tu en dis de Pierre ? il est comment ce Pierre? } \\
\text { que peut-on dire de Pierre? }\end{array}$ \\
\hline $\begin{array}{l}\text { alors finalement/ qu'est-ce que vous pensez du petit Pierre? } \\
11\end{array}, 7,10$, & $\begin{array}{l}\text { alors maintenant les enfants j'aimerais que vous me disiez ce que vous pensez de } \\
\text { maintenant/j'aimerais savoir ce que vous pensez de Pierre } \\
\text { j'aimerais bien savoir ce que vous pensez de Pierre et de son attitude } \\
\text { que penses-tu de Pierre? }\end{array}$ \\
\hline
\end{tabular}

25 À travers l'emploi de verbes d'opinion dans leur question, les enseignants semblent vouloir amener les élèves à se positionner par rapport au personnage principal. Le verbe "penser » est présent dans les questions des enseignants 3, 4, 7, 10 et 11 et le verbe « dire » apparait dans les questions des enseignants 2 et 9 . De plus, les enseignants 2 et 10 demandant de caractériser le personnage («il est comment »; «son attitude »). On peut ainsi faire l'hypothèse que, en proposant un temps de discussion partiellement décroché (Dolz \& Schneuwly, 1998) de celui de la restitution du récit, ces enseignants cherchent à créer un espace de réflexion ciblé afin de permettre aux élèves de construire une représentation plus explicite du personnage (et des raisons qui l'amènent à agir de la sorte, dans le cas des enseignants 2 et 10), qui peut contribuer à une compréhension plus fine du récit. Ce faisant, ce contenu langagier est rendu présent pour que toute la classe puisse se l'approprier (Schneuwly, 2009).

Du côté des élèves, l'analyse montre que les trois niveaux de justification présentés dans le tableau 2 peuvent apparaitre dans le corpus et avec des intensités différentes qui semblent résulter de leurs capacités langagières de compréhension mais également du type d'interventions de l'enseignant au cours des interactions en classe.

27 Ainsi, les « séances de langage » 4, 10 et 11 sont caractérisées, principalement, par une juxtaposition de propos d'élèves concernant les actions de Pierre dans le potager. On observe ainsi une (quasi)absence de prise en compte des rapports entre les actions du personnage, ses motivations et sentiments dans les justifications produites collectivement. Dans ces séances, les interventions de l'enseignant sont en entière conformité avec le «protocole de travail» de la recherche, vu qu'elles visent prioritairement la répartition de la parole entre les élèves.

Dans les séances 2, 7 et 9, sont observées des justifications très partielles mais également un début de construction plus dialectique de relations entre la caractérisation du personnage et la chronologie/logique de ses actions. Ici, la participation de l'enseignant dans le dialogue didactique reste également discrète hormis dans la séance 2 où le maitre cherche à encourager les élèves à intervenir et à reformuler leurs dires lorsqu'ils ne sont pas compris par leurs pairs. 
29 La séance 3 se distingue des précédentes et par la teneur des justifications élaborées collectivement par les élèves et par le rôle du questionnement de l'enseignant, qui favorise, dans ce cas, l'émergence de relations entre les traits pertinents concernant le système récit-personnages de Danger dans le potager.

30 Le niveau de scolarité, le type de quartier où se situe l'école ou le nombre de sessions de formation avec les membres du groupe de recherche (voir la présentation du numéro) ne semblent pas constituer des variables déterminantes pour la qualité des échanges et des justifications produites. Celles-ci sont toutefois plus systémiques dans les séances conduites par les enseignants des classes de moyenne section maternelle, qui ont, pour la plupart, suivi une quantité plus importante de sessions de formation. Il est cependant difficile de tirer des conclusions plus solides à propos de ces résultats vu le nombre restreint de séances analysées et l'absence d'informations sur la quantité de fois où le récit a été lu ou raconté aux élèves, ou encore les éventuelles activités ayant précédé chaque séance transcrite.

\section{Propos juxtaposés et traces d'une (re)construction partagée progressive du système récit-personnages de Danger dans le potager}

31 Afin d'illustrer nos résultats d'analyse, nous avons sélectionné des extraits issus des séances de langage 10 et 7 . Nous analysons ensuite des extraits de la séance 3 dans le but de les contraster avec les précédents.

Dans l'extrait sélectionné dans la séance 10, menée dans une classe de grande section hétérogène, les propos des élèves semblent se juxtaposer et leurs niveaux de justification sont presque inexistants ou très partiels surtout au début des échanges. L'analyse de la progression des interactions entre les élèves révèle toutefois des éléments dénotant une possible prise en compte des rapports entre la caractérisation du personnage principal et la chronologie/logique de ses actions.

Tableau 4. Niveaux de justification presque inexistants ou très partiels

\begin{tabular}{|l|l|l|l|}
\hline Séance & $\mathbf{N}^{\mathbf{0}}$ & Loc & Interventions \\
\hline 10 & 96 & M & $\begin{array}{l}\text { j'aimerais bien savoir ce que vous pensez de Pierre et de son attitude// } \\
\text { Ewan }\end{array}$ \\
\hline 10 & 97 & Ewan & il était plus malin que les autres \\
\hline 10 & 98 & M & Lucie \\
\hline 10 & 99 & Lucie & il est le plus intrépide de la famille \\
\hline 10 & 100 & M & Alvaro \\
\hline 10 & 101 & Alvaro & il bougeait tout le temps \\
\hline 10 & 102 & M & Ted \\
\hline 10 & 103 & Ted & il désobéit toujours il désobéit il a désobéi sa maman \\
\hline
\end{tabular}




\begin{tabular}{|l|l|l|l|}
\hline 10 & 104 & M & Ewan \\
\hline 10 & 105 & Ewan & et il écoutait pas sa maman/ il a été dans le jardin de monsieur Grégory \\
\hline 10 & 106 & M & Céline \\
\hline 10 & 107 & Céline & il a vu une chatte blanche \\
\hline 10 & 108 & M & Ewan \\
\hline 10 & 109 & Ewan & $\begin{array}{l}\text { Grégory parce que votre père est allé et il a fait un civet pour monsieur } \\
\text { Grégory }\end{array}$ \\
\hline
\end{tabular}

Suite à la question de l'enseignant, les élèves constituent, dans une sorte de tâtonnement collectif, une liste de propositions afin de caractériser Pierre et son comportement ( «il était plus malin que les autres»; «il est le plus intrépide de la famille»; «il désobéit toujours »). Cette caractérisation du personnage principal se fait de manière aléatoire et désincarnée du récit jusqu'au tour de parole 103 lorsque Ted apporte un complément à son intervention initiale en articulant le comportement de Pierre à un autre personnage (« il désobéit il a désobéi sa maman »). Ceci amène Ewan à retenir le propos de Ted tout en le reformulant («et il écoutait pas sa maman») et à associer un trait pertinent du comportement du personnage principal à l'élément de complication du récit («il a été dans le jardin de monsieur Grégory»). Céline semble saisir cette réorientation du dialogue didactique, imposée par Ewan. Cependant, elle relève un trait peu pertinent à ce stade de la reconstruction du système récit-personnages, vu que celui-ci se réfère à un moment de complication intervenant plus tardivement dans la trame narrative ( $«$ il a vu une chatte blanche »). C'est probablement la raison pour laquelle Ewan n'en tient pas compte lorsqu'il reconstitue les éléments fondant la situation initiale du récit («et la maman elle leur a dit va pas/ allez pas dans le jardin de monsieur Grégory parce que votre père est allé et il a fait un civet pour monsieur Grégory »). On peut le voir, la sélection et la reconstruction d'éléments pertinents au système récit-personnages sont, dans cet extrait, juste amorcées par les élèves, l'explicitation de rapports plus dialectiques entre les motivations, actions et sentiments des personnages étant ainsi encore à ses débuts.

Différemment, le deuxième extrait, issu de la séance de langage 7 menée dans une classe de moyenne section en zone sensible, illustre des prémisses d'une construction plus systémique des rapports entre la caractérisation du personnage et la chronologie/logique de ses actions qui s'articulent également, en partie, avec les sentiments et actions d'autres personnages.

Tableau 5. Prémisses de construction du système récit-personnages

\begin{tabular}{|l|l|l|l|}
\hline Séance & $\mathbf{N}^{\mathbf{0}}$ & Loc & Interventions \\
\hline 7 & 74 & $\mathrm{M}$ & maintenant/j'aimerais savoir ce que vous pensez de Pierre \\
\hline 7 & 75 & Cyrianne & il est méchant parce qu'il n'écoute pas/ \\
\hline
\end{tabular}




\begin{tabular}{|l|l|l|l|}
\hline 7 & 76 & Esma & $\begin{array}{l}\text { Pierre/ il écoute pas la maman et en plus il va dans la jardin de } \\
\text { monsieur Grégory/ }\end{array}$ \\
\hline 7 & 77 & Héléna & $\begin{array}{l}\text { Pierre/ il perd ses chaussures/ son manteau et après il court// sa } \\
\text { maman va pas être contente }\end{array}$ \\
\hline 7 & 78 & Esma & oh la la Pierre!/ je laisse parler Lynda \\
\hline 7 & 79 & Lynda & \\
\hline 7 & 80 & M & Eva \\
\hline 7 & 81 & Eva & $\begin{array}{l}\text { euh/ euh/ la maman/ elle donne une tisane de camomille à Pierre parce } \\
\text { qu'il est malade }\end{array}$ \\
\hline 7 & 82 & Héléna & ben oui! il a trop mangé dans le jardin de monsieur Grégory \\
\hline
\end{tabular}

On observe que, suite à la question de l'enseignant, Cyrianne essaie de caractériser Pierre («il est méchant») et de donner une justification très partielle à son comportement ("parce qu'il n'écoute pas »). Sa proposition, certes approximative ${ }^{4}$, permet aux autres élèves d'établir, collectivement, quelques liens entre ce premier trait de caractérisation du personnage et le déploiement de quelques-unes de ses actions dans la chronologie/ logique du récit, constituant ainsi un réseau de justifications partielles. Esma relève que Pierre «[...] écoute pas la maman et en plus il va dans la jardin de monsieur Grégory ». Héléna énumère des résultats ( il perd ses chaussures/ son manteau ») de l'action de courir, récurrente chez Pierre, en l'enchainant avec une nouvelle occurrence de cette action (« et après il court »). Par le tissage des rapports entre ces évènements et leurs conséquences sur les sentiments d'un autre personnage ( «sa maman va pas être contente »), l'élève effleure également des éléments en interaction avec un système dynamique plus large, constitué par une partie de l'ensemble des personnages du récit, de leurs sentiments et actions, ce qui engendre l'avancement de la trame narrative et un début de construction collective du système récit-personnages par l'esquisse de justifications presque complètes. Ainsi, ensemble, les interventions et justifications subséquentes d'Eva ( «la maman/ elle donne une tisane de camomille à Pierre parce qu'il est malade») et de Héléna («ben oui! il a trop mangé dans le jardin de monsieur Grégory») orientent le récit vers sa situation finale, avec, de surcroit, un regard interprétatif sur les raisons qui amènent Pierre à se sentir malade, pas explicitées dans le texte de B. Potter.

Même si le réseau des justifications partagées par les élèves ne restitue pas l'entièreté des rapports entre les motivations, sentiments et actions du personnage principal, il est possible, dans le présent extrait, de constater la fonction médiatrice (ou instigatrice) de la question initiale de l'enseignant, ce qui n'a pas été le cas dans le premier extrait, pour la construction de ces rapports par l'instauration d'un mode de parler distancié.

L'analyse de ces deux premiers extraits (et ceci est valable pour la presque totalité du corpus) confirme par ailleurs la présence assez discrète de l'enseignant dans le dialogue didactique conforme au «protocole de travail» établi par la recherche, mentionné précédemment. Nous pensons que ce choix didactique permet, sans doute, aux élèves d'apprendre à s'écouter et à interagir dans un dialogue collectif à partir de leurs niveaux 
de compréhension initiaux. Néanmoins, il a des conséquences sur la possibilité de la construction d'une référence partagée plus proche des significations du texte, celle-ci étant assurée par les interventions d'étayage de la part de l'enseignant, constitutives du parler scolaire. Ce type d'interventions est plus notable dans l'extrait sélectionné dans la séance 3 , analysé ci-dessous.

Rappelons que cette séance a été conduite dans une classe de moyenne section hétérogène et qu'elle se distingue des précédentes par la présence plus marquée d'indices d'émergence du système récit-personnages constitutif de Danger dans le potager. Ainsi, à nos yeux, l'extrait 3 exemplifie la construction collective progressive d'une référence partagée par la présence d'un raisonnement plus explicite et logique ponctué de marqueurs de relations causales entre des traits pertinents prélevés dans le récit par les élèves en fonction, particulièrement, des questions et interventions de l'enseignant.

Tableau 6. Émergence du système récit-personnages

\begin{tabular}{|l|l|l|l|}
\hline Séance & $\mathbf{N}^{\mathbf{0}}$ & Loc & Interventions \\
\hline 3 & 331 & M & alors finalement/ qu'est-ce que vous pensez du petit Pierre \\
\hline 3 & 332 & Melwan & que c'était bien \\
\hline 3 & 333 & Malo & il est gentil \\
\hline 3 & 334 & Viviane & quand maman elle a dit d'aller dans le pré/ il était méchant \\
\hline 3 & 335 & M & oui/ pourquoi \\
\hline 3 & 336 & Clarissa & s'il le dit il sera puni \\
\hline 3 & 337 & Viviane & parce qu'il embête monsieur Grégory \\
\hline 3 & 338 & Clarissa & parce qu'il était dans le jardin de monsieur Grégory \\
\hline 3 & 339 & Malo & il mangeait les légumes de monsieur Grégory \\
\hline
\end{tabular}

Dans le début de cet extrait, on peut tout d'abord remarquer l'établissement de relations entre des traits pertinents de caractérisation du personnage de Pierre identifiés par Malo et Viviane. Malo caractérise le personnage de manière générale et appréciative («il est gentil ») tandis que Viviane pondère (« quand maman elle a dit d'aller dans le pré/ il était méchant»), certes dans une justification partielle pleine d'implicites, sur le fait que Pierre est «méchant " (comme déjà relevé dans l'extrait 2) parce qu'il ne suit pas les conseils de Maman Lapin (et préfère aller dans le potager de monsieur Grégory). Elle cherche ainsi à caractériser le personnage de manière située, à un moment précis du récit. La proposition de l'élève amène l'enseignant à lui demander de la justifier (« oui/ pourquoi ?»), cette question rendant possible aux autres élèves de mettre en lien un ensemble de propos dans le but d'expliciter les traits de comportement du personnage principal. Ce geste didactique donne à voir les niveaux de compréhension des élèves concernant les relations dialectiques entre des éléments constitutifs du système récitpersonnages dans Danger dans le potager, à savoir, le comportement de Pierre, et les 
actions qui en découlent, vis-à-vis des injonctions maternelles ainsi que le résultat de ses actions. En examinant les justifications proposées dans les tours de parole 336 à 339, on observe que Clarissa semble être la seule à tenir compte de ces relations (justification presque complète), même si celles-ci ne sont pas entièrement explicitées («s'il le dit il sera puni »; " parce qu'il était dans le jardin de monsieur Grégory ») vu que Viviane (" parce qu'il embête monsieur Grégory ») et Malo ("il mangeait les légumes de monsieur Grégory ») déplacent leur regard vers les rapports entre les actions de Pierre et leurs possibles effets sur le comportement d'un autre personnage, le voisin.

Malgré le fait que la suite de l'extrait ouvre sur un questionnement de l'enseignant invitant à une appréciation morale du comportement de Pierre («est-ce que c'est bien?»; «pourquoi ?»), les réponses données par les élèves restent toujours ancrées dans le récit. Le réseau des justifications produites à partir du tour de parole 340 montre, toutefois, une progression dans la construction du système récit-personnages.

Tableau 7. Progression dans la construction du système récit-personnages par des justifications collectives presque complètes

\begin{tabular}{|l|l|l|l|}
\hline Séance & $\mathbf{N}^{\mathbf{0}}$ & Loc & Interventions \\
\hline 3 & 340 & M & est-ce que c'est bien \\
\hline 3 & 341 & Viviane & non \\
\hline 3 & 342 & M & pourquoi \\
\hline 3 & 343 & Clarissa & parce que monsieur Grégory il veut pas \\
\hline 3 & 344 & Malo & c'est interdit \\
\hline 3 & 345 & Melwan & et il les a mangés quand même \\
\hline 3 & 346 & Clarissa & parce que sinon les autres ils vont tuer le lapin \\
\hline 3 & 347 & Reda & ils vont manger le lapin \\
\hline 3 & 348 & Malo & et après/ il y aura plus de choux \\
\hline
\end{tabular}

Pour répondre aux questions de l'enseignant, les élèves mettent en dialogue, dans un ensemble de justifications presque complètes, les actions de Pierre, les motivations et actions de Monsieur Grégory et les conseils de Maman Lapin. En s'appuyant mutuellement sur leurs propos, Clarissa prend le point de vue de Monsieur Grégory ("parce que monsieur Grégory il veut pas »; " parce que sinon les autres ils vont tuer le lapin ») et Malo reformule ("c'est interdit») en quelque sorte les dires de sa camarade, faisant référence, de manière implicite, aux paroles de Maman Lapin. Melwan complète l'assertion de Malo ("et il les a mangés quand même») et Reda renchérit sur la proposition de Clarissa ( ils vont manger le lapin ») pour que, finalement, Malo revienne sur le point de vue du voisin (« et après/ il y aura plus de choux ») en suggérant, toujours implicitement, que celui-ci serait ainsi contrarié. 
Lorsque l'enseignant reprend son intervention initiale concernant ce que l'on peut penser ou dire de Pierre, de nouvelles réorganisations s'opèrent dans les raisonnements des élèves en lien avec ce qui précède.

Tableau 8. Une caractérisation conceptuelle plus précise du comportement du personnage

\begin{tabular}{|l|l|l|l|}
\hline Séance & $\mathbf{N}^{\mathbf{0}}$ & Loc & Interventions \\
\hline 3 & 349 & M & finalement/ que pourrait-on dire de Pierre \\
\hline 3 & 350 & Viviane & qu'il est gentil \\
\hline 3 & 351 & Yannick & il est méchant \\
\hline 3 & 352 & Clarissa & parce qu'il a désobéi \\
\hline 3 & 353 & Malo & et monsieur Grégory/ il est méchant \\
\hline
\end{tabular}

Un réseau de traits pertinents pour caractériser le personnage principal s'établit à travers les propositions de différents élèves (« il est gentil »; «il est méchant ») et aboutit ainsi à une caractérisation conceptuelle plus précise ( il a désobéi ») de son comportement. C'est peut-être la raison pour laquelle l'enseignant demande aux élèves d'articuler ce comportement aux actions de Pierre dans le tout du récit.

Tableau 9. Vers une articulation entre le comportement et les actions du personnage par la médiation enseignante

\begin{tabular}{|l|l|l|l|}
\hline Séance & $\mathbf{N}^{\mathbf{0}}$ & Loc & Interventions \\
\hline 3 & 354 & M & qu'est-ce qu'il a fait Pierre \\
\hline 3 & 355 & Malo & des bêtises \\
\hline 3 & 356 & Clarissa & il a mangé les légumes de monsieur Grégory \\
\hline
\end{tabular}

Elle cherche, également, à amener les élèves à établir des rapports entre le personnage de Pierre et celui de Maman Lapin par l'intermédiaire, cette fois-ci, d'un élément externe au récit, leur propre vécu personnel, probablement dans le but d'obtenir, finalement, des éléments de réponse aux questions posées dans les tours de parole 340 et 342.

Tableau 10. Vers des rapports entre les personnages et le vécu personnel des élèves par la médiation enseignante

\begin{tabular}{|l|l|l|l|}
\hline Séance & $\mathbf{N}^{\mathbf{0}}$ & Loc & Interventions \\
\hline 3 & 357 & M & des bêtises/ quand est-ce qu'on fait des bêtises \\
\hline 3 & 358 & Malo & quand on mange les choux de quelqu'un \\
\hline
\end{tabular}




\begin{tabular}{|l|l|l|l|}
\hline 3 & 359 & Viviane & quand on désobéit à sa maman \\
\hline 3 & 360 & Reda & c'est pas bien \\
\hline 3 & 361 & M & Viviane \\
\hline 3 & 362 & Viviane & quand on désobéit à sa maman::: \\
\hline 3 & 363 & M & donc on fait des bêtises \\
\hline 3 & 364 & Clarissa & quand on désobéit à sa maman \\
\hline 3 & 365 & Malo & sa maman de Pierre/ elle savait::: elle savait pas \\
\hline 3 & 366 & Clarissa & que Pierre était dans le jardin \\
\hline 3 & 367 & Viviane & s'il le dit/ il se fera disputer et il aura une fessée \\
\hline 3 & 368 & Yannick & il sera puni \\
\hline 3 & 369 & Yannick & il ira au coin \\
\hline
\end{tabular}

Cette intervention permet aux élèves d'identifier des traits pertinents liés aux personnages de Pierre et de sa maman dans un effort collectif de généralisation des règles de comportement social. Parfois, cette généralisation reste encore très liée au contexte du récit («quand on mange les choux de quelqu'un»). D'autres fois, les éléments de généralisation proposés dépassent les limites de la fiction et entrent en résonance avec les propres expériences de vie des élèves ("quand on désobéit à sa maman»), Ces éléments sont ensuite remobilisés dans la compréhension d'aspects moins explicités dans le texte comme les raisons qui amènent Pierre à ne pas raconter ses aventures à Maman Lapin («s'il le dit/ il se fera disputer et il aura une fessée »; «il sera puni»; «il ira au coin »), ce qui atteste des capacités des élèves à anticiper et à contrôler réciproquement ses propres hypothèses de compréhension.

Enfin, le réseau de relations entre les motivations, sentiments et actions des personnages établis dans cet extrait dénote des traces d'un processus émergent de construction de relations dynamiques, sans focalisation sur un seul aspect, entre la caractérisation du personnage de Pierre et la chronologie/logique de ses actions avec un essai de prise en compte des sentiments, motivations et actions des personnages de Monsieur Grégory et Maman Lapin. Ces relations plus ou moins complexes, qui constituent le cœur du système récit-personnages dans Danger dans le potager, restent absentes dans les extraits précédents. Comme nous l'avons dit plus haut, leur construction nous semble possible dans cette classe de moyenne section maternelle parce que l'enchainement des multiples propositions et justifications des élèves est ici systématiquement orchestré par les questions et les interventions d'étayage de l'enseignant, ce qui permet aux élèves de parler ensemble sur le récit en y développant ainsi un rapport plus distancié. 


\section{Conclusions et ouvertures possibles}

$47 \mathrm{Au}$ terme de notre contribution, nous pouvons affirmer que l'exercice d'analyse d'un corpus commun, tel qu'il a été proposé par les coordinateurs du colloque Apprentissage du langage oral à l'école maternelle. Regards croisés sur un corpus homogène, s'est révélé très stimulant. Il nous a tout d'abord donné l'opportunité, très rare dans le domaine de la recherche, de poser un regard sur des données récoltées et aménagées dans des conditions établies à partir des objectifs de recherche d'autrui. En ce sens, la tâche scientifique attribuée aux chercheurs contributeurs était doublement intéressante vu qu'elle permettait, d'une part, de prendre connaissance d'un corpus étendu constitué sur la base de présupposés théoriques-méthodologiques et de buts plus ou moins partagés, et d'autre part, de tenter de transposer les principes d'un cadrage théoriqueméthodologique propre à l'analyse de données qui leur étaient, en principe, étrangères.

Un des enjeux du colloque étant la réflexion sur l'apprentissage du langage oral à l'école maternelle, cet exercice d'analyse nous a conduite, dans un premier temps, à essayer de délimiter le statut de l'oral dans les interactions entre l'enseignant et les élèves dans les "séances de langage » composant le corpus. Cette première lecture des données nous a révélé que le dialogue scolaire caractérisant les différentes séances filmées et transcrites permettait aux élèves de développer de nouvelles capacités langagières à l'oral mais que celui-ci n'était pas érigé en tant qu'objet d'enseignement-apprentissage à part entière vu qu'il constituait, prioritairement, un médiateur des apprentissages concernant la compréhension d'un récit, un moyen pour prendre de la distance et en parler.

Ce constat nous a donc amenée, dans un deuxième temps, à essayer d'examiner le corpus, à partir de la perspective théorique-méthodologique que nous adoptons dans nos propres travaux sur la compréhension des récits portés par des albums de la littérature de jeunesse dans des classes maternelles. Nous avons ainsi cherché à analyser les démarches de justification collectives dans les échanges enseignant-élèves à propos du personnage principal et de son comportement dans le récit. Ceci dans le but de situer les niveaux de compréhension des élèves et les manières de parler ensemble, sous le guidage de l'enseignant, sur les rapports, constitutifs de la trame narrative du récit, entre les motivations, les sentiments et les actions de ce personnage. Autrement dit, d'identifier les traces de construction d'un système récit-personnages. Les résultats de l'analyse ont montré effectivement que, indépendamment du niveau scolaire et du quartier, les démarches de justifications collectives en classe peuvent rendre possible la construction progressive d'une référence partagée élèves-enseignant concernant les significations d'un texte tout en mettant en évidence le rôle fondamental de la médiation enseignante dans ce processus.

50 Si l'enseignant apparait comme une pièce maitresse dans le dialogue didactique prenant place dans "les séances de langage » examinées, les analyses réalisées dans cette contribution nous ont également conduite à réfléchir au statut des tâches habituellement proposées aux élèves à l'école maternelle autour des objets langagiers. Dans ce contexte, ce dialogue suffirait-il à la transformation des capacités langagières des élèves concernant les objets sur lesquels il porte?

51 Les recherches que nous avons menées les dernières années sur l'enseignementapprentissage de la compréhension en lecture dans le cadre de dispositifs d'ingénierie didactique élaborés en collaboration avec des enseignants tendent à montrer le contraire. 
Dans ces recherches, les apprentissages réalisés par les élèves, en l'occurrence la construction d'une représentation plus systémique des relations entre les raisons d'agir des personnages et leurs actions dans des récits, ne semble possible qu'à partir d'un tout organisé en activités de décomposition et recomposition de ce système, autrement dit, une séquence d'activités dans lesquelles pourra s'ancrer le dialogue didactique et les démarches de justifications des élèves. Ces activités, partiellement décrochées de la situation de lecture partagée d'un récit (mais fortement articulée à celle-ci), permettent à l'enseignant de rendre visibles les différentes dimensions de l'objet à apprendre (la caractérisation des personnages, la logique/chronologie de leurs actions), de conduire les élèves à les identifier, les manipuler, pour qu'ils puissent ensuite les re-signifier autrement dans la complexité de l'objet en se l'appropriant progressivement. Nous pensons, par exemple, à : des activités de reconnaissance des différents personnages, parmi des intrus, sur la base de leurs caractéristiques et leur comportement; la construction d'outils avec les élèves (tableaux, affiches) permettant l'explicitation des liens entre les motivations («ce qu'ils veulent»), les actions («ce qu'ils font») et les sentiments («ce qu'ils ressentent») des personnages; des activités de remise en ordre des images clés d'un album avec une mobilisation forte des rapports dialectiques des éléments composant le système récit-personnages (re)construit dans les activités précédentes.

Selon nous, des séquences d'activités visant la décomposition-recomposition des objets langagiers à enseigner-apprendre peuvent ainsi contribuer de manière considérable à la construction des capacités de distanciation et de généralisation, caractéristiques du parler scolaire. Par conséquent, elles constituent une voie possible pour les différents apprentissages langagiers à réaliser par l'enfant-élève, pouvant l'amener à apprendre à parler à l'école maternelle.

\section{BIBLIOGRAPHIE}

ADAM, J.-M. \& REVAZ, F. (1996). L'analyse des récits. Paris : Seuil.

BOIRON, V. (2010). « Lire des albums de littérature de jeunesse à l'école maternelle : quelques caractéristiques d'une expertise en actes ». Repères 42, p. 105-126. En ligne : https://

journals.openedition.org/reperes/254.

BOIRON, V. (2014). « Raconter et lire des récits de fiction : effets comparés sur la compréhension d'élèves de maternelle ». Repères 50, p. 83-104. En ligne : https://journals.openedition.org/ reperes $/ 778$.

BRIGAUDIOT, M. (dir.) (2000). Apprentissages progressifs de l'écrit à l'école maternelle. Paris : Hachette éducation.

BROSSARD, M. (2008). «Concepts quotidiens/concepts scientifiques : réflexions sur une hypothèse de travail ». Carrefours de l'éducation 26, p. 67-82. En ligne : https://www.cairn.info/revuecarrefours-de-l-education-2008-2-page-67.htm. 
CHARTRAND, S.-G. (2000). « Une pratique de la communication orale : la justification de ses dires ». Québec français 118, p. 46-47. En ligne : https://www.erudit.org/fr/revues/qf/2000-n118qf1197351/56060ac.pdf.

CORDEIRO, G. S. (2014). « Justifications des élèves et médiations de l'enseignante dans une tâche de compréhension en lecture d'un conte en maternelle ». Repères 50, p. 157-176. En ligne : https:// journals.openedition.org/reperes/794.

CORDEIRO, G. S. et al. (2012). « De la fonction didactique des justifications dans la construction des savoirs en lecture/compréhension et en sciences de la nature au cycle $1:$ une réflexion sur les cadres conceptuels des recherches didactiques ». In : Dorier, J.-L. Leutenegger, F. \& Schneuwly, B. (dirs), Didactique en construction, constructions des didactiques. Bruxelles : De Boeck, p. 255-279.

DOLZ, J. \& SCHNEUWLY, B. (1998). Pour un enseignement de l'oral. Initiation aux genres formels à l'école. Paris : ESF.

FLORIN, A. (1995). Parler ensemble en maternelle. La maîtrise de l'oral, l'initiation à l'écrit. Paris : Ellipses. FRANÇAIS AUJOUR'DHUI (LE). 2012. «Lecture de récits en maternelle ». 179. En ligne : https:// www.cairn.info/revue-le-francais-aujourd-hui-2012-4.htm.

GARCIA-DEBANC, C. (1996/1997). « Pour une didactique de l'argumentation orale avec des élèves de 10 ans ». Enjeux 39-40, p. 50-79.

GROSSMANN, F. (1996). Enfances de la lecture. Manières de faire, manières de lire à l'école maternelle. Berne : P. Lang.

LENTIN, L. (1975). Apprendre à parler à l'enfant de moins de 6 ans. Où ? Quand ? Comment ? Paris : Éd. ESF.

NONNON, É. (1999). «L'enseignement de l'oral et les interactions verbales en classe : champs de référence et problématiques ». Revue française de pédagogie 129, p. 87-131. En ligne : https:// www.persee.fr/doc/rfp_0556-7807_1999_num_129_1_1067.

NONNON, É. (2016). « 40 ans de discours sur l'enseignement de l'oral : la didactique face à ses questions ». Pratiques 169-170. En ligne : https://journals.openedition.org/pratiques/3115.

REUTER, Y. (1988). «L'importance du personnage ». Pratiques 60, p. 3-22. En ligne : http:// www.pratiques-cresef.com/p060_re1.pdf.

SCHNEUWLY, B. (2009). «L'objet enseigné ». In Schneuwly, B. \& Dolz, J., Des objets enseignés en classe de français. Rennes : Presses universitaires de Rennes, p. 17-28.

SÈVE, P. \& CÈBE, S. (coords) (2014). « Lire en maternelle : la lecture avant que de savoir lire ». Repères 50. En ligne : https://journals.openedition.org/reperes/747.

TAUVERON, C. (1995). Le personnage. Une clef pour la didactique du récit à l'école élémentaire. Neuchâtel : Delachaux et Niestlé.

TERWAGNE, S. \& VANESSE, M. (2008). Le récit à l'école maternelle. Lire, jouer, raconter des histoires. Bruxelles : De Boeck.

THÉVENAZ-CHRISTEN, T. (2005). Les prémices de la forme scolaire. Études d'activités langagières orales à l'école genevoise. Thèse de doctorat en sciences de l'éducation : Université de Genève.

VINCENT, G., LAHIRE, B. \& THIN, D. (1994). « Sur l'histoire et la théorie de la forme scolaire ». In : Vincent, G. (dir.), L'éducation prisonnière de la forme scolaire? Scolarisation et socialisation dans les sociétés industrielles. Lyon : Presses universitaires de Lyon, p. 11-48. 
VYGOTSKI, L. S. (1985) [1934]. Pensée et langage. Trad. du russe par par F. Sève. Paris : Éditions sociales.

\section{NOTES}

1. Pour d'autres études récentes conduites dans des classes maternelles, voir, par exemple, le numéro 179 de Français aujourd'hui (2012) sur la lecture de récits et le numéro 50 de Repères (Sève \& Cèbe, 2014) consacré à l'enseignement et à l'apprentissage de la lecture.

2. Il ne faudrait pas non plus oublier que le statut d'un personnage, sa caractérisation, sa manière de parler et de se comporter contribue également à la reconnaissance d'un récit comme appartenant à un genre donné, aspect que nous ne développerons toutefois pas ici en raison des particularités du corpus CLEA.

3. Le document contenait également un tableau proposant le découpage du texte en phrases et en "phases" narratives ainsi que des indications à l'intention des enseignants concernant des éléments du texte à aborder avec les élèves (lexique ; caractérisation et actions des personnages) relativement à ces phrases et phases. En ligne: http://www4.ac-nancy-metz.fr/ien57boulay/ IMG/doc/Danger_dans_le_potager_texte_commente_B.doc.

4. On verra plus loin dans l'analyse d'un extrait de la séance 3 que certains élèves semblent considérer Pierre comme étant "méchant » parce qu'il désobéit à sa maman. On voit ainsi des traces liées aux capacités langagières des élèves en ce qui concerne la construction de concepts et les relations entre signifiant et signifié tout comme l'importance des interactions entre élèves, point de départ pour la mise en place d'interventions d'étayage par l'enseignant par rapport à cette construction.

\section{RÉSUMÉS}

Plusieurs auteurs soulignent l'importance de la fonction didactique des démarches de justification dans les interactions enseignant-élèves pour la construction des significations concernant les textes lus ou racontés en classe. Dans cette contribution, nous essayons de montrer comment les jeunes élèves apprennent à comprendre le récit issu de l'album Danger dans le potager dans le cadre de situations didactiques qui impliquent la construction d'un dialogue didactique collectif. Nous avons examiné le corpus CLEA à partir de la perspective théoriqueméthodologique assumée dans nos propres recherches sur l'enseignement-apprentissage de la compréhension d'albums de la littérature de jeunesse en classes de maternelle. Notre but a été de situer les niveaux de compréhension des élèves et les manières de parler ensemble, sous le guidage de l'enseignant, sur les rapports entre les motivations, les sentiments et les actions des personnages, constitutifs de la trame narrative. L'analyse révèle que, indépendamment du niveau scolaire et du quartier, les démarches de justifications collectives en classe peuvent rendre possible la construction progressive d'une référence partagée élèves-enseignant concernant les significations d'un texte tout en mettant en évidence le rôle fondamental de la médiation enseignante dans ce processus.

Several authors underline the importance of the didactic function of justification settings in teacher-students' interactions o the construction of meanings regarding read or told texts in 
classroom. In this contribution, we try to show how young students learn to understand the narrative coming from the storybook Danger dans le potager within the context of didactic situations involving the construction of a collective didactic dialog. We have examined the CLEA corpus from the theoretical-methodological perspective assumed in our own research on teaching and learning how to understand children's literature storybooks in kindergarten. We have aimed at situating the students' comprehension levels and manners of talking together, under the teacher's guiding, about relations between characters' purposes, feelings and actions, which constitute the narrative frame. Analysis reveals that, regardless school level or neighbourhood, classroom collective justification settings may enable progressive construction of a teacher-students' shared reference concerning the meanings of a text and, at the same time, bring out the fundamental role of teaching mediation in this process.

INDEX

Mots-clés : enseignement-apprentissage, compréhension des récits, dialogue didactique, démarches de justification, école maternelle

Keywords : teaching and learning, comprehension of narratives, didactic dialog, justification settings, kindergarten

\section{AUTEUR}

\section{GLAIIS SALES CORDEIRO}

Faculté de psychologie et des sciences de l'éducation, Université de Genève, CH-1211 